\title{
Emerging Multidrug Resistance of Methicillin-Resistant Staphylococcus aureus in Hand Infections.
}

\author{
Rick Tosti \\ Department of Orthopaedic Surgery and Sports Medicine, Temple University School of Medicine
}

Brian T Samuelsen

Department of Orthopaedic Surgery, Mayo Clinic

Samantha Bender

Department of Orthopaedic Surgery and Sports Medicine, Temple University School of Medicine

John R Fowler

Department of Orthopaedic Surgery, University of Pittsburgh

John Gaughan

Department of Biostatistics Consulting Center, Temple University School of Medicine Follow this and additional works at: https://jdc.jefferson.edu/rothman_institute

Part of the Other Medical Specialties Commons

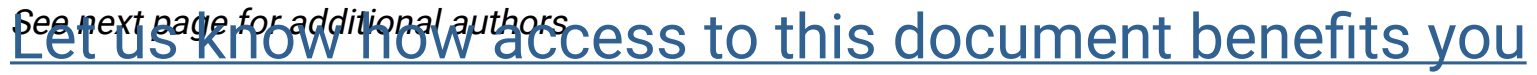

\section{Recommended Citation}

Tosti, Rick; Samuelsen, Brian T; Bender, Samantha; Fowler, John R; Gaughan, John; Schaffer, Alyssa A; and Ilyas, Asif M, "Emerging Multidrug Resistance of Methicillin-Resistant Staphylococcus aureus in Hand Infections." (2014). Rothman Institute Faculty Papers. Paper 56. https://jdc.jefferson.edu/rothman_institute/56

This Article is brought to you for free and open access by the Jefferson Digital Commons. The Jefferson Digital Commons is a service of Thomas Jefferson University's Center for Teaching and Learning (CTL). The Commons is a showcase for Jefferson books and journals, peer-reviewed scholarly publications, unique historical collections from the University archives, and teaching tools. The Jefferson Digital Commons allows researchers and interested readers anywhere in the world to learn about and keep up to date with Jefferson scholarship. This article has been accepted for inclusion in Rothman Institute Faculty Papers by an authorized administrator of the Jefferson Digital Commons. For more information, please contact: JeffersonDigitalCommons@jefferson.edu. 
Authors

Rick Tosti, Brian T Samuelsen, Samantha Bender, John R Fowler, John Gaughan, Alyssa A Schaffer, and Asif M llyas

This article is available at Jefferson Digital Commons: https://jdc.jefferson.edu/rothman_institute/56 


\title{
Emerging Multidrug Resistance of Methicillin- Resistant Staphylococcus aureus in Hand Infections
}

\author{
Rick Tosti, MD, Brian T. Samuelsen, MD, Samantha Bender, BS, John R. Fowler, MD, John Gaughan, PhD, \\ Alyssa A. Schaffer, MD, and Asif M. Ilyas, MD \\ Investigation performed at the Department of Orthopaedic Surgery and Sports Medicine, Temple University School of Medicine, \\ Philadelphia, Pennsylvania
}

\begin{abstract}
Background: Methicillin-resistant Staphylococcus aureus has been the most commonly identified pathogen in hand infections at urban centers, but the evolving antibiotic sensitivity profiles of methicillin-resistant Staphylococcus aureus are not known. The purposes of this study are to determine if multidrug resistance in methicillin-resistant Staphylococcus aureus is emerging and to provide current recommendations for empiric antibiotic selection for hand infections in endemic regions.
\end{abstract}

Methods: An eight-year longitudinal, retrospective chart review was performed on all culture-positive hand infections encountered by an urban hospital from 2005 to 2012. The proportions of all major organisms were calculated for each year. Methicillin-resistant Staphylococcus aureus infections were additionally analyzed for antibiotic sensitivity.

Results: A total of 683 culture-positive hand infections were identified. Overall, methicillin-resistant Staphylococcus aureus grew on culture in $49 \%$ of cases; the annual incidence peaked at $65 \%$ in 2007 . Over the study period, methicillinresistant Staphylococcus aureus was universally resistant to penicillin, oxacillin, and ampicillin. Clindamycin resistance significantly increased, approaching $20 \%$ by $2012(p=0.02)$. Levofloxacin resistance linearly increased from $12 \%$ to $50 \%$ $(\mathrm{p}<0.01)$. Resistance to trimethoprim-sulfamethoxazole, tetracycline, gentamicin, and moxifloxacin was only sporadically observed. Resistance to vancomycin, daptomycin, linezolid, and rifampin was not observed.

Conclusions: Significant increases in resistance to clindamycin and levofloxacin were observed in recent years, and empiric therapy with these drugs may have limited efficacy, especially in urban centers.

Clinical Relevance: Hand infections caused by methicillin-resistant Staphylococcus aureus may be developing increasing resistance to clindamycin and levofloxacin in recent years. This longitudinal study examines the effectiveness of a variety of antibiotics to methicillin-resistant Staphylococcus aureus.

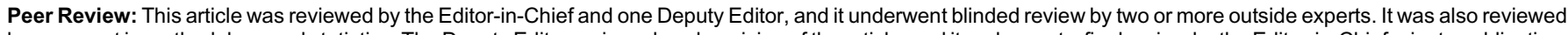

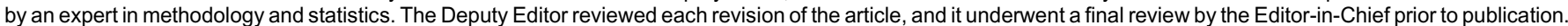
Final corrections and clarifications occurred during one or more exchanges between the author(s) and copyeditors.

S taphylococcus aureus is the most commonly cultured organism in hand infections ${ }^{1-6}$. Traditional management of acute hand abscesses has been incision and drainage followed by a regimen of beta-lactam antibiotics such as methicillin, nafcillin, or cephalosporins ${ }^{1,2}$. However, shortly following the introduction of methicillin in the 1960s, emerging strains of methicillin-resistant Staphylococcus aureus (MRSA) were reported as first occurring in the nosocomial setting and more recently in the community setting ${ }^{3-10}$. As MRSA has been more closely monitored, studies have noted associations with increased treatment failures, inpatient lengths of stay, and healthcare costs ${ }^{4,7,8,11,12}$. However, a few recent reports have suggested that treatment failures, costs, and lengths of stay may be equivalent to non-MRSA infections if an appropriate empiric antibiotic is selected $^{4,6}$.

As a result of multiple reports showing a greater prevalence of MRSA in urban settings, the Centers for Disease Control and Prevention have recommended against selecting a

Disclosure: None of the authors received payments or services, either directly or indirectly (i.e., via his or her institution), from a third party in support of any aspect of this work. One or more of the authors, or his or her institution, has had a financial relationship, in the thirty-six months prior to submission of this work, with an entity in the biomedical arena that could be perceived to influence or have the potential to influence what is written in this work. No author has had any other relationships, or has engaged in any other activities, that could be perceived to influence or have the potential to influence what is written in this work. The complete Disclosures of Potential Conflicts of Interest submitted by authors are always provided with the online version of the article. 

Volume 96-A - Number 18 -September 17, 2014
The Journal of Bone \& Joint Surgery - JBjS. Org

Multidrug Resistance in Hand Infections

TABLE I Organisms Grown on Culture from Specimens from Acute Hand Infections, 2005 to 2012

$10 \%$ to $15 \%$, and many institutions have now subsequently excluded beta-lactams from empiric treatment ${ }^{4,13,14}$. However, the efficacy of contemporary empiric drug selections is not known, and the possibility of growing multidrug resistance exists, as alternative antibiotics are becoming more commonly selected to cover MRSA. The purposes of this study are to determine if multidrug resistance in MRSA is emerging and to provide current evidence that clinicians can reference for empiric inpatient or outpatient treatment of hand infections.

\section{Materials and Methods}

A retrospective study was performed at an urban academic medical center A over an eight-year period from January 1, 2005, through December 31, 2012. After approval was obtained from the institutional review board, all hand infection cases encountered by the emergency room, outpatient office, or inpatient wards were reviewed. We identified subjects by searching International Classification of Diseases, Ninth Revision, codes relevant to hand infections, including codes $681.00,681.01,681.02,682.4,727.05,727.9,883.00,883.1$, 882.01 , and 882.00 (cellulitis, abscess, tenosynovitis, and open wounds of the fingers and hands) but only analyzed those subjects between the ages of eighteen and eighty-nine years with a culture-positive hand abscess. Demographic and laboratory data were collected from medical records. Patients with multiple culture results in the same admission were also identified so that the same organism was not counted twice. Infections were considered nosocomial if records indicated a history of a surgical procedure, dialysis treatments, catheterizations, hospitalization, or nursing home stays within a year prior to admission. We calculated the annual frequencies of culture-positive infections for the three most common isolates (MRSA, methicillin-sensitive Staphylococcus aureus [MSSA], and Streptococcus pyogenes) and polymicrobial infections. A polymicrobial infection was defined as an infection in which more than one organism was identified; these were not considered mutually exclusive with the frequencies of other organisms. MRSA infections were then further analyzed for their antibiotic sensitivity profiles. Isolates were assessed for yearly resistance rates to ampicillin, oxacillin, penicillin, clindamycin, erythromycin, levofloxacin, moxifloxacin, trimethoprim-sulfamethoxazole, tetracycline, gentamicin, rifampin, daptomycin, vancomycin, and linezolid. We also performed a post hoc calculation for MSSA resistance to clindamycin and levofloxacin.

\section{Statistical Analysis}

Continuous variables were assessed with a linear regression model. Percentages of categorical variables and drug resistances were assessed for a significant increasing or decreasing trend with the Cochrane-Armitage trend test. Significance was defined as $\mathrm{p}<0.05$.

\section{Source of Funding}

No external source of funding was used for this study.

\section{Results}

\section{Overall Demographic Characteristics}

A total of 683 culture-positive hand infections were identi$A$ fied over the ninety-six-month collection period. The average patient age was 41.4 years, and $56 \%$ were male; the average white blood cell count was $10.8 \times 10^{3} / \mu \mathrm{L}$. The most common etiologies for cases were trauma $(75 \%)$ and intravenous drug use $(21 \%)$; bite wounds were causative in $3 \%$ of cases. The average annual frequency of comorbidities was consistent over the study period for patients with diabetes (14.6\% [range, $12.6 \%$ to $28.6 \%$ ] of the population) and for patients who were positive for the human immunodeficiency

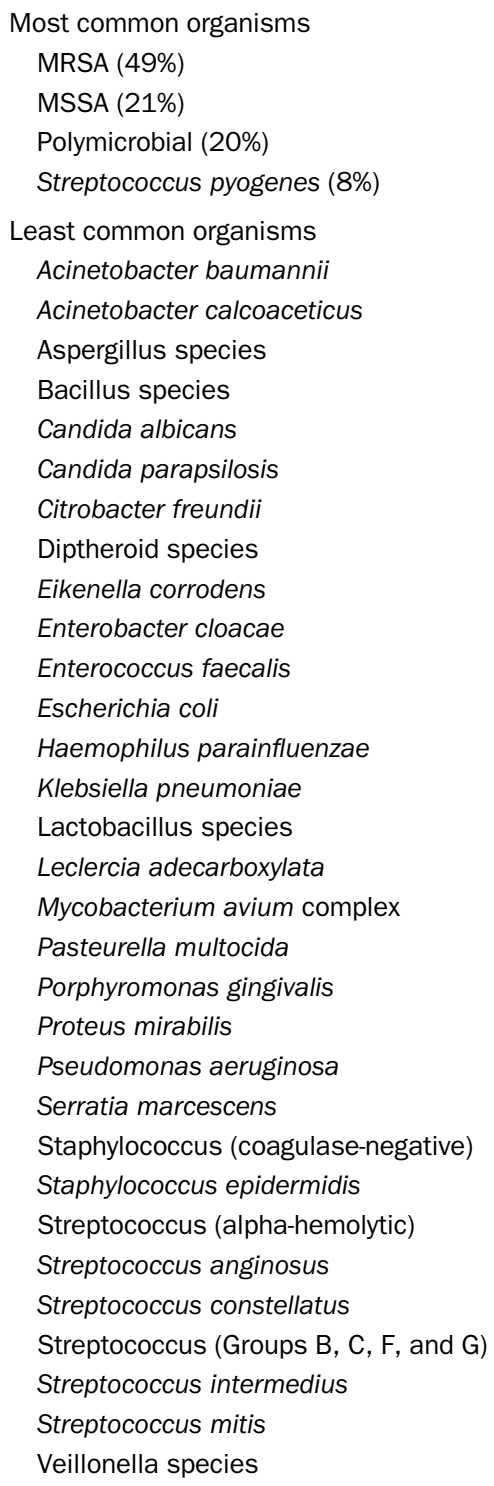

virus (3.3\% [range, $0 \%$ to $5.7 \%$ ] of the population). However, intravenous drug users represented a larger percentage of people with culture-positive hand infections over the time span of the study: $13.8 \%$ in 2005 and $37.2 \%$ in 2012 ( $\mathrm{p}<0.01$ ). Patients with a history of cancer or nursing home residence were each noted in approximately $2 \%$ of all hand infections. MRSA infections were considered to be community acquired in $76 \%$ of cases and nosocomial in $24 \%$ of cases.

\section{Organisms Grown on Culture per Annum}

Overall, MRSA was the organism most commonly grown on culture, followed by MSSA and then Group A Streptococcus. A variety of organisms were found in lesser frequencies (Table I). MRSA was also the most common pathogen identified during 
The Journal of Bone \& Joint Surgery $\cdot$ Jbjs.org Volume 96-A - Number $18 \cdot$ September 17, 2014

TABLE II Annual Resistance of MRSA to Selected Antibiotics

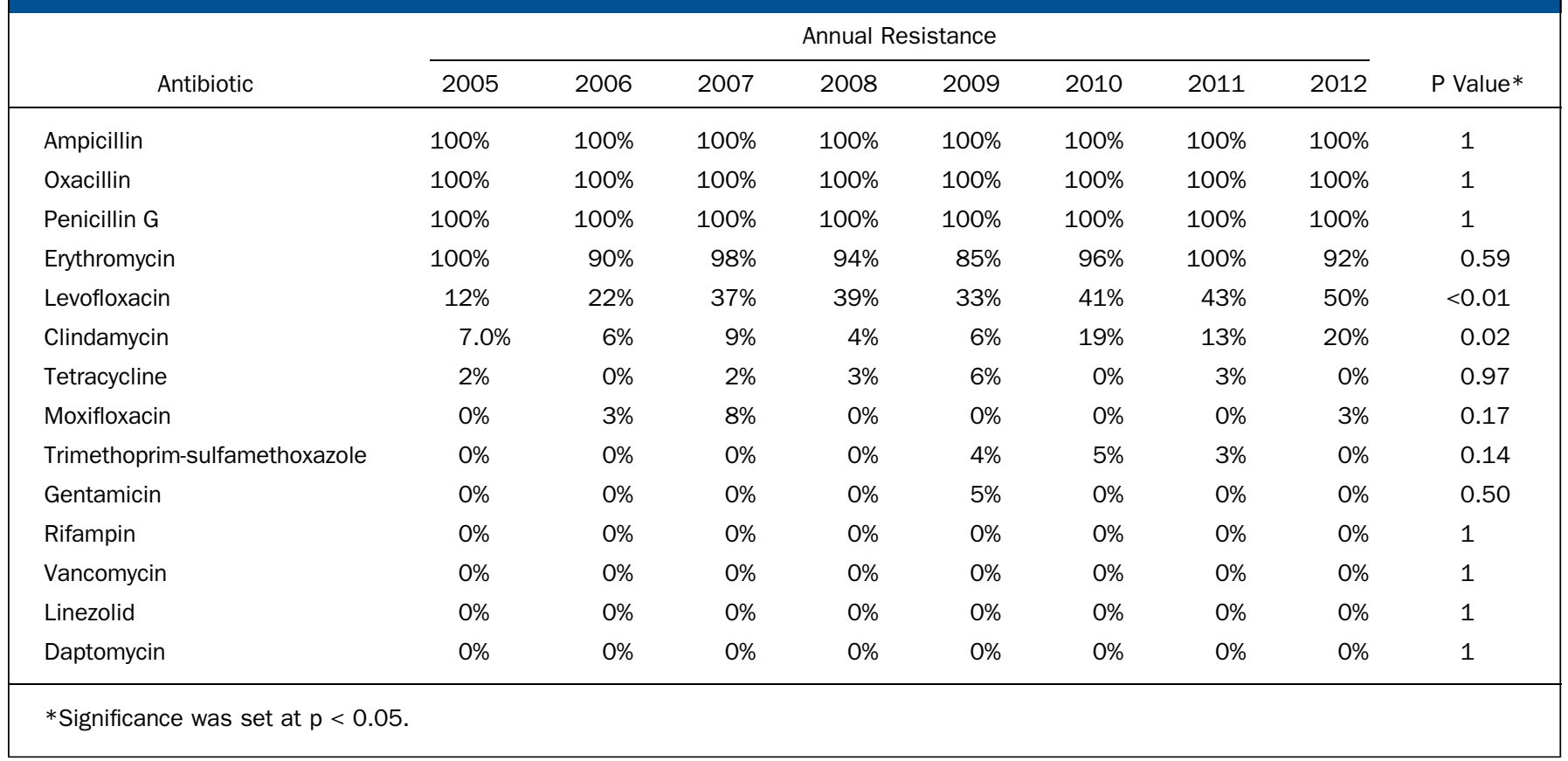

every year of the study, and it grew on culture in the following proportions: $53 \%$ in $2005,63 \%$ in $2006,65 \%$ in $2007,43 \%$ in $2008,47 \%$ in $2009,45 \%$ in $2010,37 \%$ in 2011 , and $42 \%$ in 2012 (Fig. 1). The number of MSSA infections fluctuated inversely, with MRSA reaching a nadir in 2006 and 2007 but increasing to a peak of $27.5 \%$ in 2012 . A significant increase $(\mathrm{p}<0.01)$ was observed in the percentage of polymicrobial infections, as they comprised $7 \%$ in $2005,16 \%$ in $2006,13 \%$ in $2007,30 \%$ in $2008,31 \%$ in $2009,36 \%$ in $2010,37 \%$ in 2011 , and $25 \%$ in 2012. Streptococcus pyogenes infections were grown on culture in $6 \%$ to $17 \%$ of cases per year.

For intravenous drug users and diabetic patients with hand infections, MRSA remained the most common organism each year, reaching a peak incidence in 2007 as well. Intravenous drug users had the highest annual percentages of MRSA of all subgroups. Diabetic patients with hand infections commonly had polymicrobial infections (33\% overall [range, $22 \%$ to $50 \%$ per year]), but this percentage did not increase over

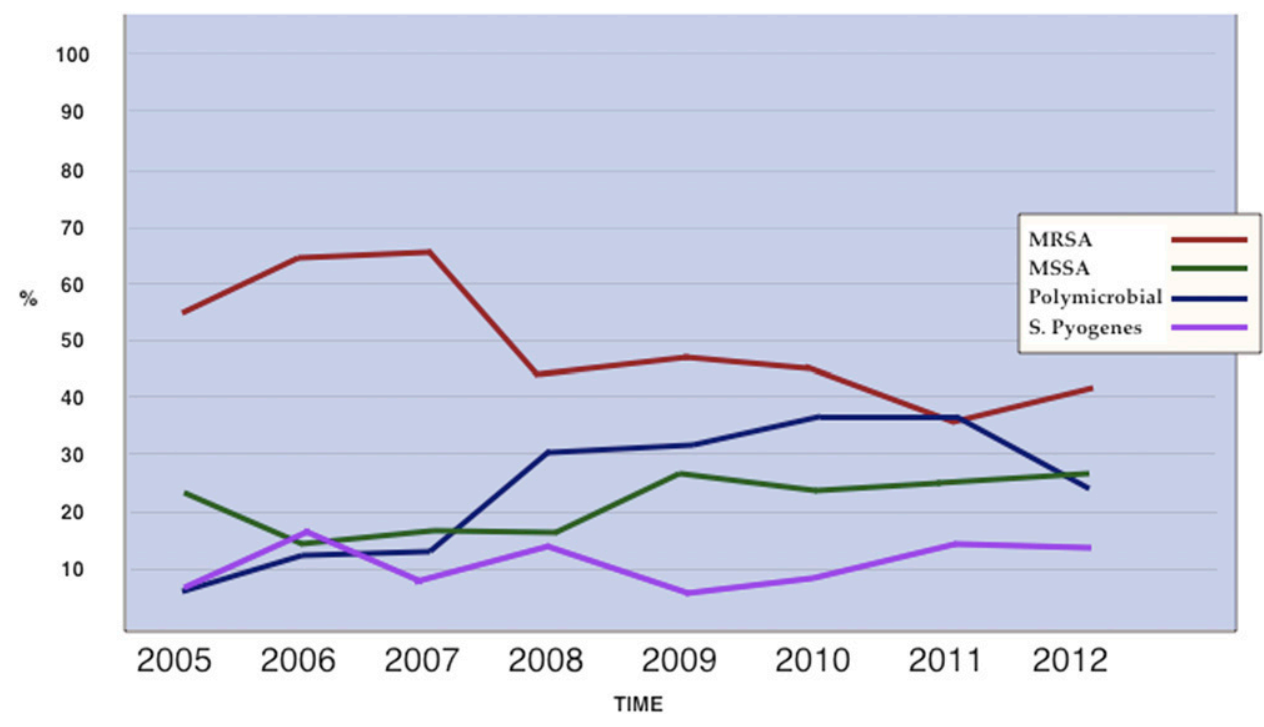

Fig. 1

The annual percentages of MRSA, MSSA, polymicrobial organisms, and Streptococcus pyogenes grown on culture from specimens from acute hand infections. 
The Journal of Bone \& Joint Surgery $\cdot$ JbJs.org Volume 96-A - Number 18 - September 17, 2014
Multidrug Resistance in Hand Infections

in approximately half of all hand infections in urban communities in recent years. Moreover, increased costs, failures in treatment, and increased mortality have also been associated with MRSA in endemic regions $\mathrm{s}^{4,6-9,11,12}$; however, multiple studies from our institution have shown that equivalent lengths of hospital stay to non-MRSA infections can be achieved if appropriate empiric antibiotic coverage is selected ${ }^{4,6}$. As MRSA continues to predominate in urban communities, we sought to further characterize its antibiotic sensitivities to optimize the empiric treatment regimen, to reduce treatment delays, and to improve cost containment associated with hand infections.

MRSA grown on culture from specimens from hand infections was increasingly resistant to clindamycin and levofloxacin over the eight-year period, and the sensitivity to other antibiotics did not appear to change. In recent years, MRSA resistance to clindamycin has approached $20 \%$ at our center. Although not directly answered by the present study, we suspect that the increased use of clindamycin to treat MRSA may be contributory. In 2005, we adopted a hand infection algorithm to improve the coverage of MRSA, which largely excluded beta-lactams from empiric treatment of hand infections ${ }^{4,8}$. Therapeutics such as vancomycin, clindamycin, and trimethoprim-sulfamethoxazole were used with a much greater frequency, and, in particular, clindamycin was the most commonly prescribed initial antibiotic for hand infections, representing $40 \%$ to $50 \%$ of the empiric selections from 2005 to 2007.

In addition, levofloxacin resistance linearly increased over the past eight years in our study. A report by MacDougall et al. noted a significant association between emerging MRSA infections and quinolone prescriptions in a cross-sectional study of seventeen regional U.S. hospitals; the authors suggested that quinolones may be driving a selection process for MRSA, but their conclusions were limited in that the MRSA resistance to in hand abscesses ranging from $34 \%$ to $73 \% \%^{4,12-18}$. Our results agree with prior studies and indicate that MRSA may be found

\section{Discussion}

$\mathrm{M}$ RSA has gained attention in the literature and in popular media as a growing concern, especially in urban environments, as several reports have estimated a high proportion

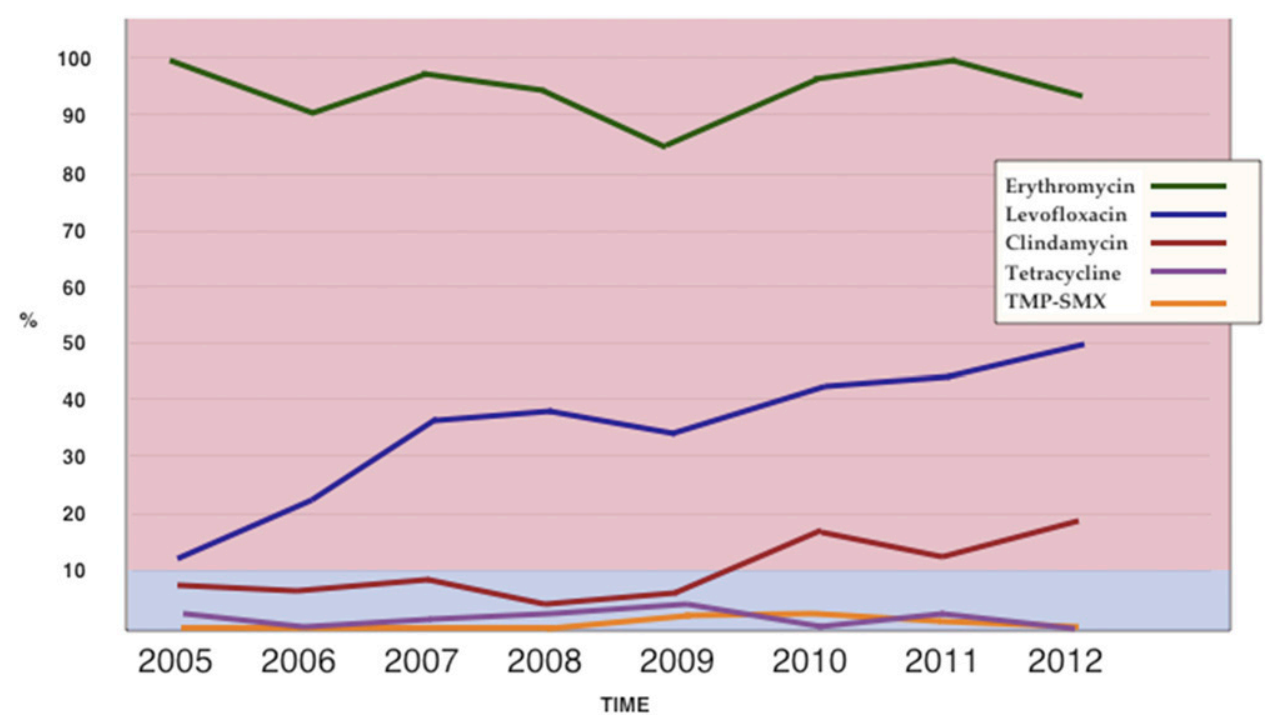

Fig. 2

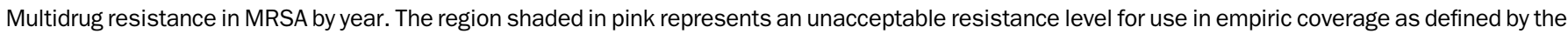
Centers for Disease Control and Prevention. Antibiotics found to have $100 \%$ resistance or sensitivity are not displayed. TMP-SMX $=$ trimethoprimsulfamethoxazole. 
The Journal of Bone \& Joint Surgery $\cdot$ Jbjs.org Volume 96-A - Number $18 \cdot$ September 17,2014
Multidrug Resistance in Hand Infections

still appear to have potent activity against MRSA. Organisms that grew on culture from specimens from polymicrobial infections were often sensitive to most antibiotics, and we would not recommend empiric treatment for anaerobic or gram-negative bacteria unless specific situations such as bite wounds, aquatic injuries, or severe infections warranted such coverage. In this series, infections caused by bite wounds were generally susceptible to beta-lactam antibiotics, which may be selected in an empiric management protocol under these circumstances. Finally, we would encourage future studies evaluating the efficacy of common empiric antibiotics in other soft-tissue and joint infections of the extremities with interval monitoring to ensure relevant and current management recommendations.

Rick Tosti, MD

Samantha Bender, BS

John Gaughan, $\mathrm{PhD}$

Alyssa A. Schaffer, MD

Department of Orthopaedic Surgery and Sports Medicine

(R.T., S.B., and A.A.S.) and Biostatistics Consulting Center (J.G.),

Temple University School of Medicine,

3401 North Broad Street,

Philadelphia, PA 19140.

E-mail address for R. Tosti: rtosti@temple.edu

Brian T. Samuelsen, MD

Department of Orthopaedic Surgery,

Mayo Clinic, 200 First Street S.W.,

Rochester, MN 55905

John R. Fowler, MD

Department of Orthopaedic Surgery,

University of Pittsburgh,

Kaufmann Building,

3471 Fifth Avenue, Suite 911,

Pittsburgh, PA 15213

Asif M. Ilyas, MD

Rothman Institute,

Thomas Jefferson University,

925 Chestnut Street,

Philadelphia, PA 19107

\section{References}

1. Kanavel AB. The classic. Infections of the hand. Clin Orthop Relat Res. 1974 Oct;(104):3-8.

2. Houshian S, Seyedipour S, Wedderkopp N. Epidemiology of bacterial hand infections. Int J Infect Dis. 2006 Jul;10(4):315-9. Epub 2006 Feb 17.

3. Daum RS. Clinical practice. Skin and soft-tissue infections caused by methicillinresistant Staphylococcus aureus. N Engl J Med. 2007 Jul 26;357(4):380-90.

4. O'Malley M, Fowler J, llyas AM. Community-acquired methicillin-resistant Staphylococcus aureus infections of the hand: prevalence and timeliness of treatment. J Hand Surg Am. 2009 Mar;34(3):504-8.

5. Fowler JR, llyas AM. Epidemiology of adult acute hand infections at an urban medical center. J Hand Surg Am. 2013 Jun;38(6):1189-93. Epub 2013 May 3.

6. Fowler JR, Greenhill D, Schaffer AA, Thoder JJ, llyas AM. Evolving incidence of MRSA in urban hand infections. Orthopedics. 2013 Jun:36(6):796-800.

7. Shorr AF. Epidemiology and economic impact of meticillin-resistant Staphylococcus aureus: review and analysis of the literature. Pharmacoeconomics. 2007;25(9):751-68.
8. Shorr AF. Epidemiology of staphylococcal resistance [Review]. Clin Infect Dis. 2007 Sep 15;45(Suppl 3):S171-6.

9. Karanas YL, Bogdan MA, Chang J. Community acquired methicillin-resistant Staphylococcus aureus hand infections: case reports and clinical implications. J Hand Surg Am. 2000 Jul;25(4):760-3.

10. Connolly B, Johnstone F, Gerlinger T, Puttler E. Methicillin-resistant Staphylococcus aureus in a finger felon. J Hand Surg Am. 2000 Jan;25(1):173-5.

11. Lodise TP Jr, McKinnon PS. Burden of methicillin-resistant Staphylococcus aureus: focus on clinical and economic outcomes. Pharmacotherapy. 2007 Jul;27(7):1001-12.

12. Downs DJ, Wongworawat MD, Gregorius SF. Timeliness of appropriate antibiotics in hand infections. Clin Orthop Relat Res. 2007 Aug;461(461):17-9.

13. Kiran RV, McCampbell B, Angeles AP, Montilla RD, Medina C, Mitra A, Gaughn J, Spears J, Mitra A. Increased prevalence of community-acquired methicillin-resistant Staphylococcus aureus in hand infections at an urban medical center. Plast Reconstr Surg. 2006 Jul;118(1):161-6; discussion 167-9. 
The Journal of Bone \& Joint Surgery - JBjs.org Volume 96-A · Number $18 \cdot$ SePtember 17,2014
Multidrug Resistance in Hand Infections

Escherichia coli in 17 US hospitals. Clin Infect Dis. 2005 Aug 15;41(4):435-40. Epub 2005 Jun 28.

14. Tosti R, llyas AM. Empiric antib
Surg Am. 2010 Jan;35(1):125-8.

15. Bach HG, Steffin B, Chhadia AM, Kovachevich R, Gonzalez MH. Communityassociated methicillin-resistant Staphylococcus aureus hand infections in an urban setting. J Hand Surg Am. 2007 Mar;32(3):380-3.

16. Wilson PC, Rinker $B$. The incidence of methicillin-resistant staphylococcus aureus in community-acquired hand infections. Ann Plast Surg. 2009 May;62(5):513-6.

17. LeBlanc DM, Reece EM, Horton JB, Janis JE. Increasing incidence of methicillinresistant Staphylococcus aureus in hand infections: a 3-year county hospital experience. Plast Reconstr Surg. 2007 Mar;119(3):935-40.

18. Akdemir $O$, Lineaweaver $W$. Methicillin-resistant Staphylococcus aureus hand infections in a suburban community hospital. Ann Plast Surg. 2011 May;66(5):486-7.

19. MacDougall C, Powell JP, Johnson CK, Edmond MB, Polk RE. Hospital and community fluoroquinolone use and resistance in Staphylococcus aureus and
20. Stevanovich M, Sharpe F. Acute infections. In: Wolfe SW, Hotchkiss RN, Pederson WC, Kozin SH, editors. Green's Operative Hand Surgery. 6th ed. Philadelphia: Churchill Livingstone; 2011. p 45.

21. Moran GJ, Krishnadasan A, Gorwitz RJ, Fosheim GE, McDougal LK, Carey RB, Talan DA; EMERGEncy ID Net Study Group. Methicillin-resistant S. aureus infections among patients in the emergency department. N Engl J Med. 2006 Aug 17;355(7): 666-74.

22. Gorwitz RJ, Jernigan DB, Powers JH, Jernigan JA, and Participants in the CDCConvened Experts' Meeting on Management of MRSA in the Community. Strategies for clinical management of MRSA in the community: summary of an experts' meeting convened by the CDC. 2006. http://www.cdc.gov/mrsa/pdf/MRSA-StrategiesExpMtgSummary-2006.pdf. Accessed 2014 Mar 26. 